\title{
OPEN Ultrahigh efficient spin orbit torque magnetization switching in fully sputtered topological insulator and ferromagnet multilayers
}

\author{
Tuo Fan ${ }^{1}$, Nguyen Huynh Duy Khang ${ }^{1,2}$, Soichiro Nakano ${ }^{1}$ \& Pham Nam Hai ${ }^{1,3,4 凶}$
}

Spin orbit torque (SOT) magnetization switching of ferromagnets with large perpendicular magnetic anisotropy has a great potential for the next generation non-volatile magnetoresistive random-access memory (MRAM). It requires a high performance pure spin current source with a large spin Hall angle and high electrical conductivity, which can be fabricated by a mass production technique. In this work, we demonstrate ultrahigh efficient and robust SOT magnetization switching in fully sputtered BiSb topological insulator and perpendicularly magnetized Co/Pt multilayers. Despite fabricated by the magnetron sputtering instead of the laboratory molecular beam epitaxy, the topological insulator layer, BiSb, shows a large spin Hall angle of $\vartheta_{\mathrm{SH}}=10.7$ and high electrical conductivity of $\sigma=1.5 \times 10^{5}$ $\Omega^{-1} \mathrm{~m}^{-1}$. Our results demonstrate the feasibility of BiSb topological insulator for implementation of ultralow power SOT-MRAM and other SOT-based spintronic devices.

Embedded non-volatile memories have great impacts on energy-efficient electronics, including Internet-ofThing, Artificially Intelligent (AI), among others. To be successful, non-volatile memories have to satisfy several requirements, such as high writing endurance, high capacity, high speed, and low fabrication cost. Among many emerging non-volatile memory technologies, magnetoresistive random-access memory (MRAM) is one of the most promising that have gained development commitment from several leading semiconductor companies. The latest MRAM technology using the sophisticated spin-transfer-torque (STT) writing technique has just been commercially available very recently, but already found various important applications, such as highly efficient AI chips. However, in STT-MRAM, a large writing current has to be injected directly to magnetic tunneling junctions (MTJs), which leads to reliability issues such as accelerated aging of the oxide tunnel barrier ${ }^{1}$. In addition, large writing currents require large driving transistors, making it difficult to increase the bit density of STT-MRAM beyond the 1 Gbit capacity. Recently, the spin-orbit-torque (SOT) technique has emerged as a promising writing method for the next generation MRAM ${ }^{2}$. In SOT-MRAM, a charge current flowing in a non-magnetic layer with large spin-orbit interaction can generate a pure spin current by the spin Hall effect. The pure spin current is then injected to the magnetic free layer for magnetization switching. The relationships between the spin current $I_{\mathrm{S}}$ and the charge current $I_{\mathrm{C}}$ is given by $I_{\mathrm{S}}=(\hbar / 2 e)(L / t) \theta_{\mathrm{SH}} I_{\mathrm{C}}$, where $L$ is the MTJ size, and $t$ the thickness of the spin Hall layer, and $\theta_{\mathrm{SH}}$ is the spin Hall angle. Theoretically, the charge-to-spin conversion efficiency $(L / t) \theta_{\mathrm{SH}}$ in SOT-MRAM can be larger than unity, meaning that lower driving currents can be expected. Furthermore, since there is no current flowing into the MTJs, reliability can be significantly improved. Finally, since the spin-polarization of the pure spin current is perpendicular to the magnetization direction of the free magnetic layer, the spin torque is maximized and the magnetization can switch very fast $(<\mathrm{ns})$ in SOT-MRAM with perpendicular magnetic anisotropy (PMA) ${ }^{3}$. Because of those merits of SOT-MRAM comparing with STTMRAM, there have been huge efforts to find spin Hall materials with large $\theta_{\mathrm{SH}}$ and high electrical conductivity for SOT-MRAM implementation. Heavy metals, such as $\mathrm{Pt}^{4,5}, \mathrm{Ta}^{2}$, and $\mathrm{W}^{6}$, have been studied extensively as candidates for the spin Hall layer in SOT-MRAM, since they have been used in STT-MRAM manufacturing. However, $\theta_{\mathrm{SH}}$ of heavy metals is of the order of $\sim 0.1$, and the typical critical switching current density in heavy metals/

\footnotetext{
${ }^{1}$ Department of Electrical and Electronic Engineering, Tokyo Institute of Technology, 2-12-1 Ookayama, Meguro, Tokyo 152-8550, Japan. ${ }^{2}$ Department of Physics, Ho Chi Minh City University of Education, 280 An Duong Vuong Street, District 5, Ho Chi Minh City 738242, Vietnam. ${ }^{3}$ Center for Spintronics Research Network (CSRN), The University of Tokyo, 7-3-1 Hongo, Bunkyo, Tokyo 113-8656, Japan. ${ }^{4}$ CREST, Japan Science and Technology Agency, 4-1-8 Honcho, Kawaguchi, Saitama 332-0012, Japan. ${ }^{\circledR}$ email: pham.n.ab@m.titech.ac.jp
} 
ferromagnet bilayers with PMA is of the order of $10^{7} \mathrm{Acm}^{-2}$ to $10^{8} \mathrm{Acm}^{-2}$, which is too high for Si electronics ${ }^{7}$ Thus, finding a material with large $\theta_{\mathrm{SH}}$ of the order of 10 is of emergent need.

Recently, very large $\theta_{\mathrm{SH}}(>1)$ has been observed in topological insulators (TIs), making them very promising for SOT-MRAM ${ }^{8,9}$. TIs are quantum materials having gaped bulk states but Dirac-like metallic surface states ${ }^{10-12}$ with spin-momentum locking ${ }^{13,14}$, owning to their large spin-orbit interaction and band structure topology. Although the origin of large $\theta_{\mathrm{SH}}$ of TIs was a controversial topic due to the coexistence of the bulk current and surface current ${ }^{15}$, it has been clearly shown to originate from the surface states rather than the bulk states in carefully controlled experiments ${ }^{16}$. However, in most TI thin films, the limited surface density of states restricts the electrical conductivity $\sigma$ to the order of $\sim 10^{4} \Omega^{-1} \mathrm{~m}^{-1}$ (for example, $\sigma \sim 5.7 \times 10^{4} \Omega^{-1} \mathrm{~m}^{-1}$ for $\mathrm{Bi}_{2} \mathrm{Se}_{3}$ and $1.8 \times 10^{4}$ $\Omega^{-1} \mathrm{~m}^{-1}$ for $\left.\left(\mathrm{Bi}_{0.07} \mathrm{Sb}_{0.93}\right)_{2} \mathrm{Te}_{3}\right)$, which results in a large shunting current when in contact with other metallic layers. Second, most TI thin films studied so far are single crystalline films grown epitaxially by the laboratory molecular beam epitaxy (MBE) technique on dedicated III-V semiconductor substrates, which is not compatible with mass production. Furthermore, ultralow power SOT magnetization switching at room-temperature with current densities of the order of $10^{5}-10^{6} \mathrm{Acm}^{-2}$ have been demonstrated mostly in TI/magnetic layers whose magnetic anisotropy is very small, such as in $\mathrm{Bi}_{2} \mathrm{Se}_{3} / \mathrm{NiFe}$ bilayer with nearly zero in-plane magnetic anisotropy ${ }^{17}$, or in a $\mathrm{Bi}_{2} \mathrm{Se}_{3} / \mathrm{CoTb}$ bilayer with a small magnetization of $200 \mathrm{emu} / \mathrm{cc}$ and a PMA field of less than $2 \mathrm{kOe}^{18}$. For TI being a practical material for SOT-MRAM, the following three minimum requirements must be satisfied: (1) a large spin Hall angle of the order of 10, (2) large electrical conductivity $\sigma$ of order of $10^{5} \Omega^{-1} \mathrm{~m}^{-1}$, and (3) can be deposited using sputtering deposition. The first two requirements are for optimization of the switching current density and switching energy (see Suppl. Info. Section 1). The closest attempt is $\mathrm{Bi}_{\mathrm{x}} \mathrm{Se}_{1-\mathrm{x}}$ thin films deposited by the sputtering technique, which shows a promisingly large $\theta_{\mathrm{SH}}=8.7-18.6$ but with the expense of reduced electrical conductivity $\left(\sigma \sim 7.8 \times 10^{3} \Omega^{-1} \mathrm{~m}^{-1}\right)^{19}$. Thus, the three requirements for a practical spin Hall material for SOT-MRAM have not yet been achieved.

Among various TI material candidates, $\mathrm{Bi}_{1-x} \mathrm{Sb}_{x}(0.07<x<0.22)$ is the best hope. BiSb is the first discovered three-dimensional TI with small bulk bandgap $(\sim 20 \mathrm{meV})$ and high bulk conductivity $\sigma$ of $4-6.4 \times 10^{5}$ $\Omega^{-1} \mathrm{~m}^{-113,14,20}$. In thin films, the quantum size effect significantly increases the band gap of BiSb, so that the current flows mostly on the surface when the thickness reaches $10 \mathrm{~nm}^{21}$. Thanks to the multi-surface states, $\sigma$ of BiSb thin films is as high as $2.5 \times 10^{5} \Omega^{-1} \mathrm{~m}^{-1}$. Furthermore, a giant spin Hall effect with $\theta_{\mathrm{SH}} \sim 52$ has been observed in $\mathrm{BiSb}(012)$ thin films in junctions with MnGa grown on GaAs substrates by $\mathrm{MBE}^{22}$. Nevertheless, following works on sputtered polycrystalline BiSb yields a maximum $\theta_{\mathrm{SH}}$ of only $1.2 \sim 2.4$ (Refs. ${ }^{23,24}$ ), or even no spin Hall effect $^{25}$. Therefore, it is very important to demonstrate the three requirements for sputtered BiSb for any realistic applications to SOT-based spintronic devices.

In this work, we demonstrate ultrahigh efficient SOT magnetization switching in fullly sputtered $\mathrm{BiSb}-(\mathrm{Co} /$ Pt) multilayers with large PMA. We show that the sputtered BiSb has a large spin Hall angle of $\theta_{\mathrm{SH}}=10.7$ and high electrical conductivity of $\sigma=1.5 \times 10^{5} \Omega^{-1} \mathrm{~m}^{-1}$, thus satisfying all the three requirements for SOT-MRAM implementation. Despite the large PMA field of $5.2 \mathrm{kOe}$ of the $(\mathrm{Co} / \mathrm{Pt})$ multilayers, we achieve robust SOT magnetization at a low current density of $1.5 \times 10^{6} \mathrm{Acm}^{-2}$. Our results demonstrate the potential of BiSb topological insulator for ultralow power SOT-MRAM and other SOT-based spintronic devices.

\section{High quality topological insulator-perpendicularly magnetized ferromagnetic multilayers}

The first step in this study is to demonstrate that it is possible to use the sputtering deposition technique for fabrication of high-quality topological insulator and perpendicularly magnetized ferromagnetic multilayers. Considering the large surface roughness of TIs ( 5-6 Angstrom for BiSb) and atomic interdiffusion during the annealing process of the MTJ, it is not realistic to deposit the MTJ with large PMA on top of the TI layer. Instead, the MTJ should be deposited and fabricated first, then the TI layer should be deposited on top of the free magnetic layer at the last step. Furthermore, to achieve high enough thermal stability, the free magnetic layer should be composed of ferromagnetic multilayers with high PMA that couple ferromagnetically or antiferromagnetically to the $\mathrm{CoFeB}$ layer ${ }^{26}$. Noting that the $(\mathrm{Co} / \mathrm{Pt})_{\mathrm{n}}(\mathrm{n}=2-6)$ multilayers have been frequently used in MRAM production as a part of the synthetic antiferromagnetic reference layer with large PMA for pinning, we chose the $(\mathrm{Co} 0.4 \mathrm{~nm} / \mathrm{Pt} 0.4 \mathrm{~nm})_{2}$ multilayers (referred below as CoPt) to realize thin ferromagnetic multilayers with large PMA for evaluating the SOT performance of the BiSb layer (see Suppl. Info. Section 2).

Figure 1a shows the studied multilayer heterostructure, which consists of perpendicularly magnetized (Co $0.4 \mathrm{~nm} / \mathrm{Pt} 0.4 \mathrm{~nm})_{2}$ multilayers/10 $\mathrm{nm} \mathrm{Bi}_{0.8} \mathrm{Sb}_{0.15}$ topological insulator layer, capped by $1 \mathrm{~nm} \mathrm{MgO} / 1 \mathrm{~nm} \mathrm{Pt}$ (not shown). The multilayers were deposited on $c$-plane sapphire substrates by a combination of direct current (DC) and radio-frequency (RF) magnetron sputtering in a multi-cathode chamber. Characterization by X-ray diffraction and transmission electron microscopy shows that the BiSb layer is highly textured with the dominant (110) orientation (see Suppl. Info. Section 3). We also evaluated several sputtered BiSb single layers on sapphire substrates and observed the existence of metallic surface states as well as insulating bulk states with a band gap more than $170 \mathrm{meV}$ for thin $(<20 \mathrm{~nm})$ BiSb films ${ }^{27}$.

For electrical measurements, we fabricated $25 \mu \mathrm{m}$-wide Hall bars by optical lithography. An optical image of a Hall bar device with the experiment setup is shown in Fig. 1b. From the parallel resistor model, we estimate that the conductivity of the BiSb layer is $1.5 \times 10^{5} \Omega^{-1} \mathrm{~m}^{-1}$, which is much higher than that of sputtered $\mathrm{Bi}_{1-x} \mathrm{Se}_{x}$, and close to that of MBE grown BiSb on GaAs(111)A substrates. This demonstrates that is possible to grow highly conductive BiSb topological thin films on top of perpendicularly magnetized metallic layers by the sputtering technique. Thanks to the high conductivity of the BiSb layer, $50 \%$ of the applied current flows into the BiSb and contribute to the SOT magnetization switching. 
(a)

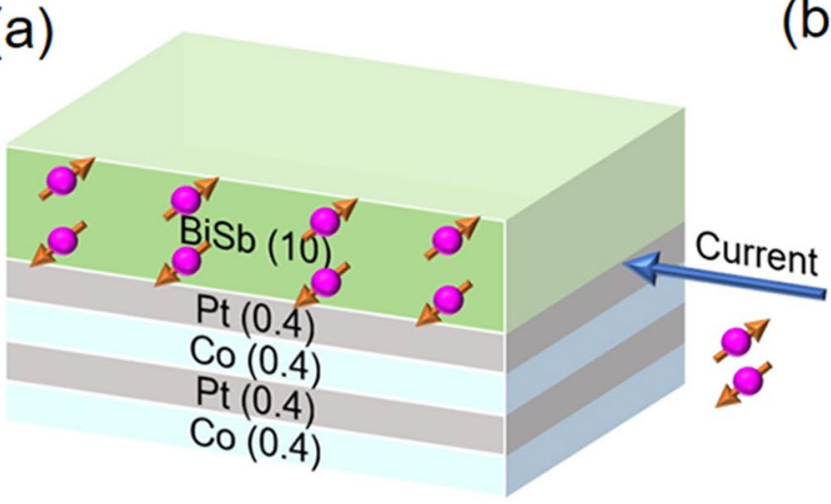

(b)

(c)

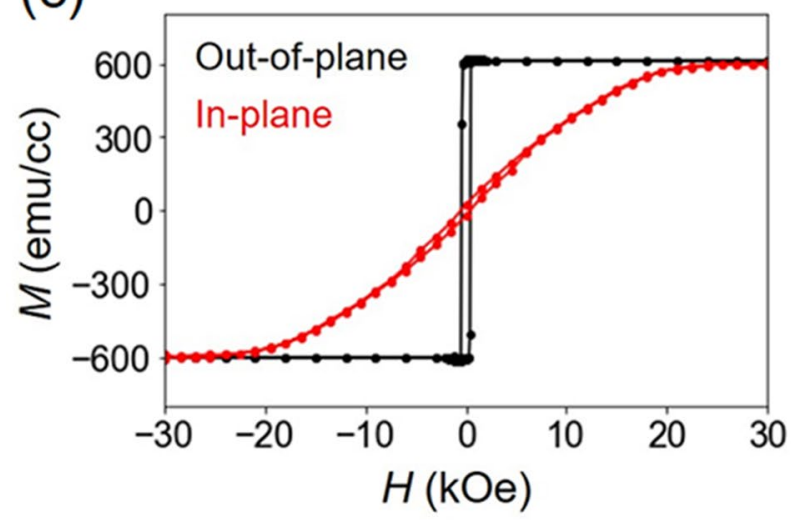

(d)

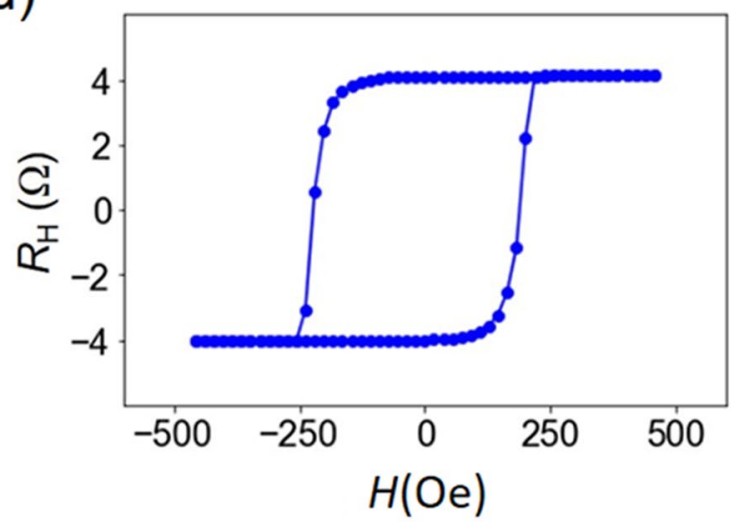

Figure 1. Perpendicularly magnetized Co/Pt ferromagnetic multilayers - topological insulator BiSb heterostructure. (a) Schematic structure of our multilayers. (b) Optical image of a Hall bar device and measurement configuration. (c) Magnetization curves of the Co/Pt multilayers. (d) Hall resistance of a Hall bar device measured with a perpendicular magnetic field.

Figure 1c shows the magnetic hysteresis curves of the as-grown sample measured by a superconducting quantum interference device (SQUID) under an in-plane and out-of-plane external magnetic field, respectively. The saturation magnetization $M_{\mathrm{CoPt}}$, normalised by the CoPt layer thickness $\left(t_{\mathrm{CoPt}}=1.6 \mathrm{~nm}\right)$, is $613 \mathrm{emu} \cdot \mathrm{cm}^{-3}$. The uniaxial anisotropy field $H_{\mathrm{k}}=15 \mathrm{kOe}$ is very large for the as-grown film. After Hall bar device fabrication by optical lithography undergoing several circles of thermal annealing, $H_{\mathrm{k}}$ is reduced to $5.2 \mathrm{kOe}$ (see Suppl. Info. Section 4), but is still much larger than that of NiFe or CoTb used in previous works. This CoPt layer yields a thermal stability factor for magnetization switching of $\Delta=38$, similar to that of the $\mathrm{CoFeB}(/ \mathrm{MgO})$ free layer of perpendicular MTJs with diameters of $20-40 \mathrm{~nm}^{28,29}$. Figure $1 \mathrm{~d}$ shows the anomalous Hall resistance $\left(R_{\mathrm{H}}\right)$ measured for a Hall bar under a sweeping out-of-plane field, which confirms PMA of the CoPt layer.

\section{Evaluation of the spin Hall angle by the second harmonic Hall measurements}

Next, we performed the second harmonic Hall measurements to evaluate the spin Hall angle ${ }^{30}$. An alternating current (AC) $J=J_{0} \sin \omega \mathrm{t}(\omega=259.68 \mathrm{~Hz})$ was applied to the Hall bar under a sweeping external field along the $x$ direction. We measured the 2 nd harmonic Hall resistance $R_{\mathrm{H}}^{2 \omega}$, which is originated from the oscillation of the net magnetic moment under the spin-orbit effective magnetic fields ${ }^{31-33}$. Figure 2 a shows a representative $R_{\mathrm{H}}^{2 \omega}-H_{x}$ curve measured at $J^{\mathrm{BiSb}}=3.6 \times 10^{5} \mathrm{Acm}^{-2}$. The second harmonic Hall resistance $R_{\mathrm{H}}^{2 \omega}$ can be expressed as ${ }^{33}$

$$
R_{\mathrm{H}}^{2 \omega}=\frac{R_{\mathrm{H}}}{2} \frac{H_{\mathrm{AD}}}{H_{x}-H_{\mathrm{k}}\left(H_{x} /\left|H_{x}\right|\right)}+R_{\mathrm{PHE}} \frac{H_{\mathrm{FL}+\mathrm{Oe}}}{H_{x}}+R_{\text {thermal }} \frac{H_{x}}{\left|H_{x}\right|}
$$

where $H_{\mathrm{AD}}$ is the antidamping-like effective field, $H_{\mathrm{FL}+\mathrm{Oe}}$ is the sum of the fieldlike and Oesterd field, $R_{\mathrm{PHE}}$ is the planar Hall resistance, and $R_{\text {thermal }}$ is the contribution from the anomalous Nernst (ANE) and spin Seebeck (SSE) effects. Note that the contribution of the fieldlike and Oesterd field to $R_{\mathrm{H}}^{2 \omega}$ is much smaller than that of the antidampinglike field in $\mathrm{BiSb}^{34,35}$. Fitting Eq. (1) to the high field data in the $R_{\mathrm{H}}^{2 \omega}-H_{x}$ curve yields $H_{\mathrm{AD}}$ (red curves in Fig. 2a). Figure $2 \mathrm{~b}$ shows $H_{\mathrm{AD}}$ as a function of $J^{\mathrm{Bisb}}$. From the $H_{\mathrm{AD}} / J^{\mathrm{Bisb}}$ gradient, we can calculate the effective spin Hall angle $\theta_{\mathrm{SH}}^{\mathrm{eff}}=\frac{2 e}{\hbar} M_{\mathrm{CoPt}} t_{\mathrm{CoPt}} \frac{H_{\mathrm{AD}}}{J^{\mathrm{BiSb}}}=12.3$, where $e$ is the electron charge and $\hbar$ is the reduced Plank constant. Recently, it was reported that CoPt multilayers can generate a "self" spin-orbit torque ${ }^{36}$. We found that the "self" spin-orbit torque in CoPt can contribute to $13 \%$ of the total spin-orbit torque (see Suppl. Info. Section 6). Furthermore, we confirmed that there is no artifact contribution from the asymmetric magnon scattering mechanism 

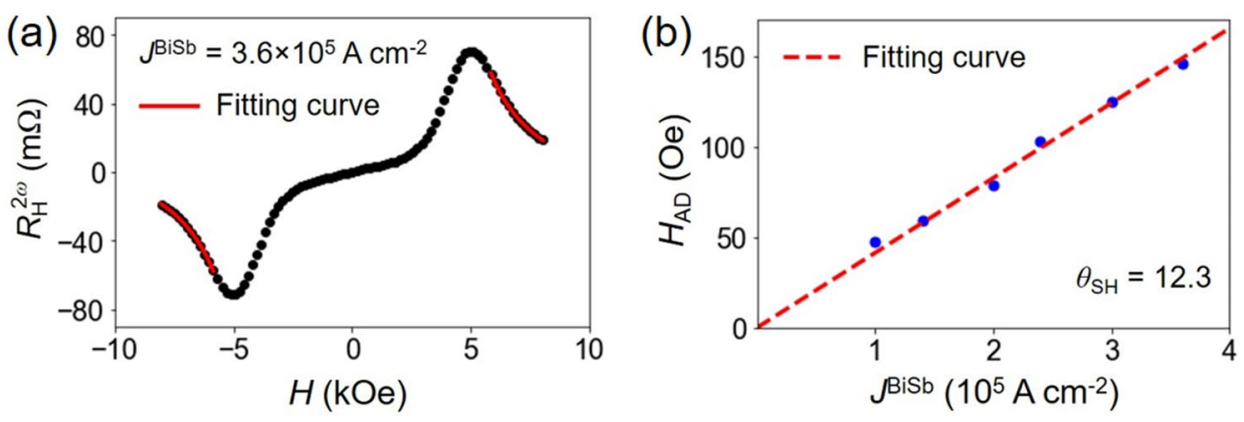

Figure 2. Evaluation of the spin Hall angle by the second harmonic measurements. (a) 2nd harmonic Hall resistance as a function of the in-plane external magnetic field $H$ applied along the $x$ direction. The red curves are the theoretical fitting using Eq. (1). (b) $H_{\mathrm{AD}}$ as a function of $J^{\mathrm{Bisb}}$.
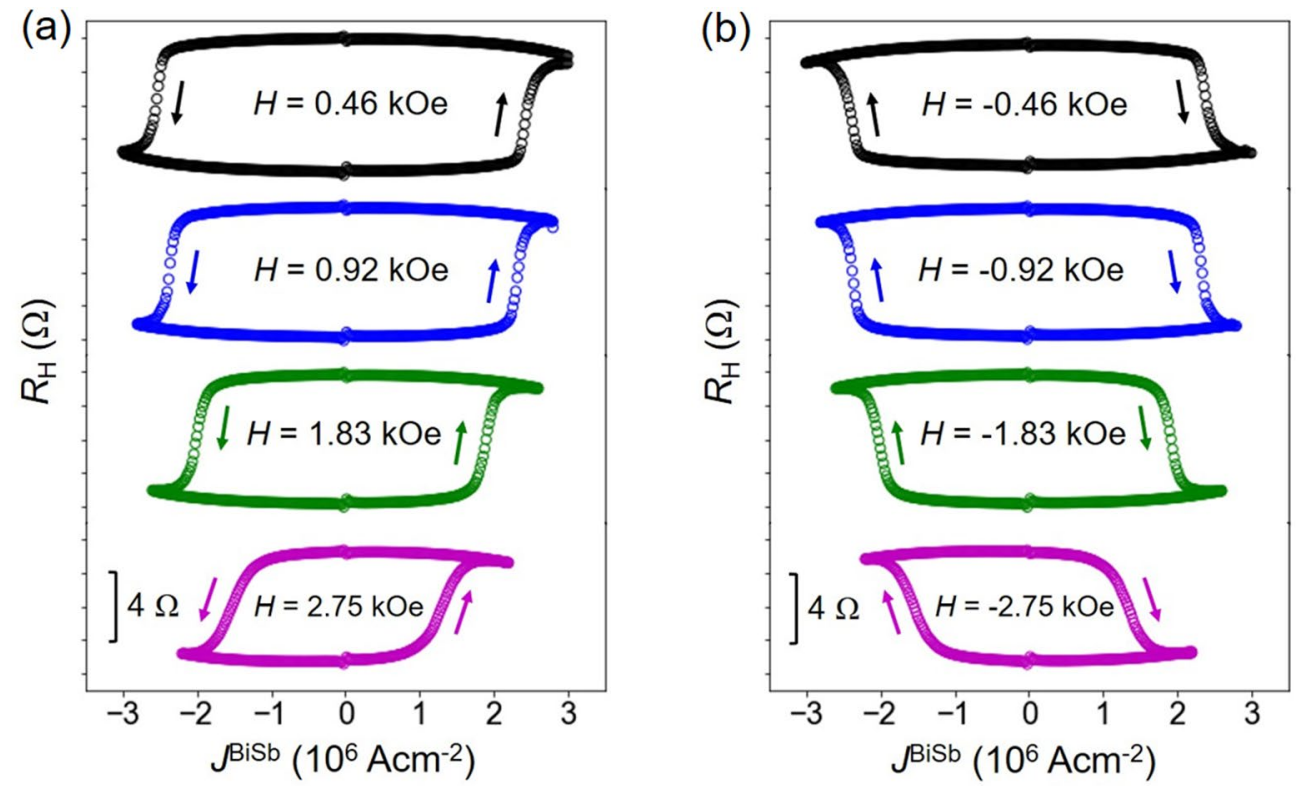

Figure 3. SOT magnetization switching by DC currents. Switching loops measured under an in-plane magnetic field applied along $(\mathbf{a})+x$ direction and $(\mathbf{b})-x$ direction.

(see Suppl. Info. Section 7), as observed in the case of the magnetic topological insulator $(\mathrm{CrBiSb})_{2} \mathrm{Te}_{3}{ }^{37}$. Subtracting the contribution from CoPt, we obtain the intrinsic spin Hall angle of $\mathrm{BiSb} \theta_{\mathrm{SH}}=10.7$, which demonstrates the feasibility of BiSb for ultralow power SOT-MRAM.

\section{Ultrahigh efficient spin-orbit torque magnetization switching by DC and pulse currents}

Next, we demonstrate ultrahigh efficient and robust SOT magnetization switching in the CoPt/BiSb multilayers. Figure 3 shows the SOT magnetization switching by DC currents with an applied external field along the $x$ direction. We achieved Hall resistance switching whose amplitude is consistent with that of the Hall resistance loop shown in Fig. 1d, indicating full magnetization switching. The switching direction is reversed when the external magnetic field direction is reversed, which is consistent with the characteristic of SOT. Typical DC threshold switching current density $J_{\mathrm{th}}^{\mathrm{BiSb}}$ is $1.5 \times 10^{6} \mathrm{Acm}^{-2}$ at the bias field of $2.75 \mathrm{kOe}$. Note that thanks to the high electrical conductivity $\sigma=1.5 \times 10^{5} \Omega^{-1} \mathrm{~m}^{-1}$ of BiSb, the total current density including the shutting current in the CoPt is kept at $2.6 \times 10^{6} \mathrm{Acm}^{-2}$. Next, we performed SOT magnetization switching by pulse currents. Figure $4 \mathrm{a}$, b show representative SOT switching loops by $0.1 \mathrm{~ms}$ pulse currents at $+1.83 \mathrm{kOe}$ and $-1.83 \mathrm{kOe}$, respectively. Figure $4 \mathrm{c}$ plots $J_{\text {th }}^{\text {BiSb }}$ at various pulse width $t_{\text {pulse }}$, and the theoretical fitting using the thermal activation model $J_{\text {th }}^{\text {Bisb }}=J_{0}^{\text {BiSb }}$ $\left.\times 1-\frac{1}{\Delta} \ln \left(\frac{\tau_{\text {pulse }}}{\tau_{0}}\right)\right]^{38}$, where $J_{0}^{B i S b}$ is the zero-kelvin threshold switching current density, $\Delta$ is the thermal stability factor, and $1 / \tau_{0}=1 \mathrm{GHz}\left(\tau_{0}=1 \mathrm{~ns}\right)$ is the attempt switching frequency. The fitting yields $J_{0}^{\mathrm{BiSb}}=4.6 \times 10^{6} \mathrm{Acm}^{-2}$ and $\Delta=38$. Because magnetization switching occurs by domain wall nucleation and domain wall motion, $\Delta$ reflects the energy barrier of the volume with size equal to the domain wall width, i.e. $\Delta$ should be considered 
(a)

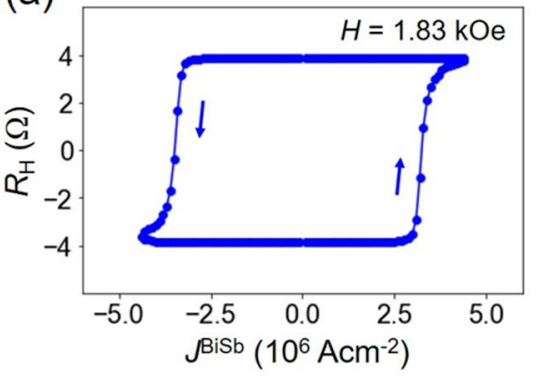

(b)

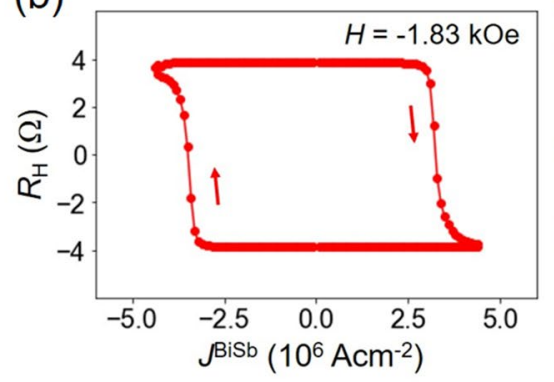

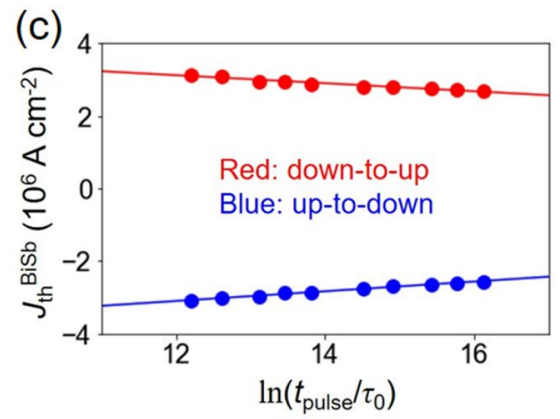

(d)
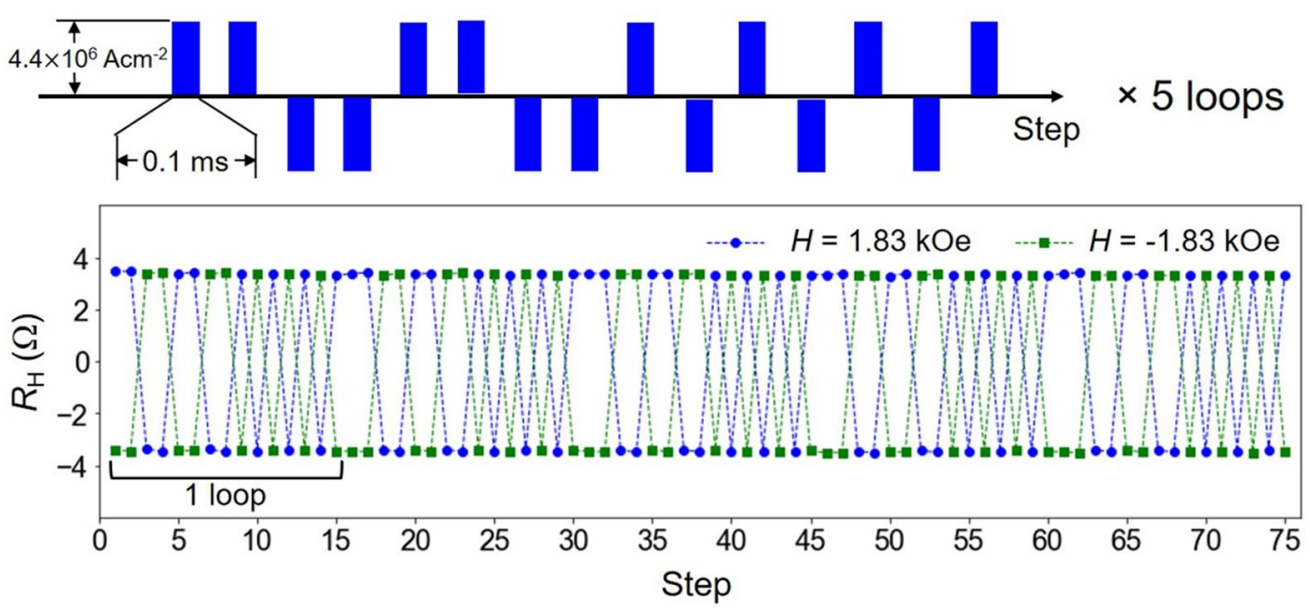

Figure 4. SOT magnetization switching by pulse currents. (a,b) Switching loop by $0.1 \mathrm{~ms}$ pulse currents under an in-plane magnetic field of $H=+1.83 \mathrm{kOe}$ and $-1.83 \mathrm{kOe}$, respectively. (c) Threshold current density $J_{\mathrm{th}}^{\mathrm{Bisb}}$ as a function of $t_{\text {pulse }}$ (d) Robust SOT magnetization switching by $0.1 \mathrm{~ms}$ pulse current.

\begin{tabular}{|l|c|l|l|l|}
\hline SOT materials & $\left|\boldsymbol{\theta}_{\mathrm{SH}}\right|$ & $\boldsymbol{\sigma}\left(\boldsymbol{\Omega}^{-1} \mathbf{m}^{-1}\right)$ & $\left|\boldsymbol{\sigma}_{\mathrm{SH}}\right|\left[(\boldsymbol{h} / \mathbf{2 e}) \boldsymbol{\Omega}^{-1} \mathbf{m}^{-1}\right]$ & $\boldsymbol{P}_{\mathbf{n}}$ \\
\hline $\mathrm{Ta}$ & 0.15 & $5.3 \times 10^{5}$ & $8.0 \times 10^{4}$ & 1 \\
\hline $\mathrm{Pt}$ & 0.08 & $4.2 \times 10^{6}$ & $3.4 \times 10^{5}$ & $3.6 \times 10^{-1}$ \\
\hline $\mathrm{W}$ & 0.4 & $4.7 \times 10^{5}$ & $1.9 \times 10^{5}$ & $1.6 \times 10^{-1}$ \\
\hline$\left(\mathrm{Bi}_{0.07} \mathrm{Sb}_{0.93}\right)_{2} \mathrm{Te}_{3}(\mathrm{MBE})$ & 2.5 & $1.8 \times 10^{4}$ & $4.5 \times 10^{4}$ & $3.0 \times 10^{-1}$ \\
\hline $\mathrm{Bi}_{2} \mathrm{Se}_{3}(\mathrm{MBE})$ & 3.5 & $5.7 \times 10^{4}$ & $2.0 \times 10^{5}$ & $2.1 \times 10^{-2}$ \\
\hline $\mathrm{Bi}_{\mathbf{x}} \mathrm{Sei}_{1-\mathrm{x}}$ (sputtered) & 18.6 & $7.8 \times 10^{3}$ & $1.5 \times 10^{5}$ & $2.6 \times 10^{-2}$ \\
\hline $\mathrm{Bi}_{0.85} \mathrm{Sb}_{0.15}$ (sputtered) & 10.7 & $1.5 \times 10^{5}$ & $1.6 \times 10^{6}$ & $5.2 \times 10^{-4}$ \\
\hline
\end{tabular}

Table 1. Spin Hall angle $\theta_{\mathrm{SH}}$, electrical conductivity $\sigma$, spin Hall conductivity $\sigma_{\mathrm{SH}}$, and SOT normalized power consumption $P_{\mathrm{n}}$ of several heavy metals and topological insulators.

as the energy barrier to nucleate a domain wall, rather than the energy barrier for coherently switching of the whole volume of the magnetic layer ${ }^{39}$. Therefore, $\Delta$ evaluated by this way is smaller than that should be expected for switching the whole volume of the magnetic layer. Nevertheless, the obtained $\Delta$ of $\mathrm{CoPt}$ is large enough to ensure that the total $\Delta$ in ferromagnetically (antiferromagnetically) coupled $\mathrm{CoFeB} / \mathrm{Ta}(\mathrm{Ru}) / \mathrm{CoPt}$ free layer can exceeds 60 for 10 years thermal stability, while the switching current density remains the same $e^{40}$. Finally, we demonstrate robust SOT switching in the CoPt/BiSb junction. For this purpose, we applied a sequence of 150 pulses $\left(J_{\text {th }}^{\mathrm{BiSb}}= \pm 4.4 \times 10^{6} \mathrm{Acm}^{-2}, t_{\text {pulse }}=0.1 \mathrm{~ms}\right)$ as shown in the top panel of Fig. $4 \mathrm{c}$. The Hall resistance data recorded for a total of 150 pulses under $\pm 1.83 \mathrm{kOe}$ are shown in the bottom panel in Fig. $4 \mathrm{c}$. We observed a robust SOT switching with no change in the device characteristics, indicating that the BiSb topological insulator deposited by the sputtering technique has great potential for realistic SOT-MRAM.

\section{SOT performance benchmarking and future prospective}

Table 1 summarizes $\theta_{\mathrm{SH}}, \sigma$, the spin Hall conductivity $\sigma_{\mathrm{SH}}=(\hbar / 2 e) \sigma \theta_{\mathrm{SH}}$, and the SOT normalized power consumption $P_{\mathrm{n}}$ at room temperature of several heavy metals and TIs. Here, $\theta_{\mathrm{SH}}$ of TIs are their best values reported in literature. For the calculation of the $P_{\mathrm{n}}$, we assumed bilayers of spin Hall material (thickness $t=6 \mathrm{~nm}$ for 
heavy metals and $t=10 \mathrm{~nm}$ for TIs) and CoFeB (thickness $t_{\mathrm{FM}}=1.5 \mathrm{~nm}$, conductivity $\sigma_{\mathrm{FM}}=6 \times 10^{5} \Omega^{-1} \mathrm{~m}^{-1}$ ). Considering the shunting current in the ferromagnetic layer, the SOT power consumption is proportional to $\left(\sigma t+\sigma_{\mathrm{FM}} t_{\mathrm{FM}}\right) /\left(\sigma t \theta_{\mathrm{SH}}\right)^{2}$. One can see that not only $\theta_{\mathrm{SH}}$ but also $\sigma$ affect the SOT power consumption, a fact usually overlooked in literature. For example, while the sputtered $\mathrm{Bi}_{x} \mathrm{Se}_{1-x}$ has a much larger spin Hall angle $\left(\theta_{\mathrm{SH}}=18.6\right)$ than that $\left(\theta_{\mathrm{SH}}=3.5\right)$ of $\mathrm{MBE}$-grown $\mathrm{Bi}_{2} \mathrm{Se}_{3}$, their power consumption is nearly the same, because $\mathrm{Bi}_{x} \mathrm{Se}_{1-x}$ has poorer crystal quality than $\mathrm{Bi}_{2} \mathrm{Se}_{3}$ and thus very low conductivity. Meanwhile, the sputtered BiSb thin film in this work shows both high $\sigma=1.5 \times 10^{5} \Omega^{-1} \mathrm{~m}^{-1}$ and large $\theta_{\mathrm{SH}}=10.7$, which are optimal for both small switching current density and small switching power consumption ${ }^{41}$. Indeed, the switching power consumption for sputtered $\mathrm{BiSb}$ is 50 times smaller than that for sputtered $\mathrm{Bi}_{x} \mathrm{Se}_{1-x}$, and over 300 times smaller than that for $\mathrm{W}$, which is the most used heavy metal in SOT-MRAM development. The small switching current density and switching power also help suppress failure of the spin Hall layer due to electromigration and Joule heating ${ }^{42}$. Our results demonstrate the feasibility of BiSb for not only ultralow power SOT-MRAM but also other SOT-based spintronic devices, such as race-track memories ${ }^{43}$ and spin Hall oscillators ${ }^{44,45}$.

Finally, we discuss about the remaining challenges for realization of ultralow power BiSb-based SOT-MRAM. One of material challenges is the large surface roughness of $\mathrm{BiSb}$ due to the unusually large crystal grain size comparing with the layer thickness. Furthermore, atomic interdiffusion between BiSb and the free magnetic layer during annealing process of the MTJ is also a challenge to be resolved. Thereby, a realistic pathway to integrate BiSb to SOT-MRAM is to fabricate full stack MTJs with PMA (p-MTJ) first, then deposit the BiSb layer on top of the MTJs at the last step after the annealing process, i.e. BiSb-on-MTJ structure. This will help avoid the interdiffusion and the BiSb surface roughness problems in the MTJ-on-BiSb structure.

\section{Method}

Material growth. We deposited multilayers of $(0.4 \mathrm{~nm} \mathrm{Co} / 0.4 \mathrm{~nm} \mathrm{Pt})_{2} / 10 \mathrm{~nm} \mathrm{Bi}{ }_{0.85} \mathrm{Sb}_{0.15} / 1 \mathrm{~nm} \mathrm{MgO} /$ $1 \mathrm{~nm}$ Pt on $c$-plane sapphire substrates by DC (for $\mathrm{Co}, \mathrm{Pt}, \mathrm{BiSb}$ ) and $\mathrm{RF}$ (for $\mathrm{MgO}$ ) magnetron sputtering in a multi-cathode chamber. All layers are deposited by sputtering from their single targets using Ar plasma without breaking the vacuum at room temperature.

Device fabrication. The samples were patterned into $90 \mu \mathrm{m}$-long $\times 25 \mu \mathrm{m}$-wide Hall bars by optical lithography and lift-off. A $45 \mathrm{~nm}$-thick Pt were deposited as electrodes by DC magnetron sputtering, which reduces the effective length of the devices to $50 \mu \mathrm{m}$.

SOT characterization. The samples were mounted inside a vacuumed cryostat equipped with an electromagnet. For the second harmonic measurements, a NF LI5650 lock-in amplifier was employed to detect the first and the second harmonic Hall voltages under sine wave excitation generated by a Keithley 6221 AC/DC current source. For the DC (pulse) current-induced SOT magnetization switching, a Keithley 2400 SourceMeter (6221 AC/DC current source) was used, and the Hall signal was measured by a Keithley 2182A NanoVoltmeter.

\section{Data availability}

The data that support this study results are available from the corresponding author upon reasonable request.

Received: 5 August 2021; Accepted: 7 February 2022

Published online: 22 February 2022

\section{References}

1. Zhao, W. S. et al. Failure and reliability analysis of STT-MRAM. Microelectron. Reliab. 52, 1848 (2012).

2. Liu, L. et al. Spin-torque switching with the giant spin Hall effect of tantalum. Science 336, 555-558 (2012).

3. Garello, K. et al. Ultrafast magnetization switching by spin-orbit torques. Appl. Phys. Lett. 105, 212402 (2014).

4. Miron, I. M. et al. Perpendicular switching of a single ferromagnetic layer induced by in-plane current injection. Nature 476, 189-193 (2011).

5. Liu, L., Lee, O. J., Gudmundsen, T. J., Ralph, D. C. \& Buhrmam, R. A. Current-induced switching of perpendicularly magnetized magnetic layers using spin torque from the spin Hall effect. Phys. Rev. Lett. 109, 096602 (2012).

6. Hao, Q. \& Xiao, G. Giant Spin Hall effect and switching induced by spin-transfer torque in a W/Co40Fe40B20/MgO structure with perpendicular magnetic anisotropy. Phys. Rev. Appl. 3, 034009 (2015).

7. Garello K. et al. Manufacturable 300mm platform solution for Field-Free Switching SOT-MRAM. 2019 Symposium on VLSI Technology, JFS4-5 (2019).

8. Mellnik, A. R. et al. Spin-transfer torque generated by a topological insulator. Nature 511, 449-451 (2014).

9. Fan, Y. et al. Magnetization switching through giant spin-orbit torque in a magnetically doped topological insulator heterostructure. Nat. Mater. 13, 699-704 (2014).

10. Bernevig, B. A., Hughes, T. L. \& Zhang, S. C. Quantum spin Hall effect and topological phase transition in HgTe quantum wells. Science 314, 1757-1761 (2006).

11. König, M. et al. Quantum spin Hall insulator state in HgTe quantum wells. Science 318, 766-770 (2007).

12. Zhang, H. et al. Topological insulators in $\mathrm{Bi}_{2} \mathrm{Se}_{3}, \mathrm{Bi}_{2} \mathrm{Te}_{3}$ and $\mathrm{Sb}_{2} \mathrm{Te}_{3}$ with a single Dirac cone on the surface. Nat. Phys. 5, 438-442 (2009).

13. Hsieh, D. et al. Observation of unconventional quantum spin textures in topological insulators. Science 323, 919-922 (2009).

14. Hirahara, T. et al. Topological metal at the surface of an ultrathin $\mathrm{Bi}_{1-\mathrm{x}} \mathrm{Sb}_{\mathrm{x}}$ alloy film. Phys. Rev. B 81, 165422 (2010).

15. Hai, P. N. Spin Hall effect in topological insulators. J. Magn. Soc. Jpn. 44, 137-144 (2020).

16. Wu, H. et al. Room-temperature spin-orbit torque from topological surface states. Phys. Rev. Lett. 123, 207205 (2019).

17. Wang, Yi. et al. Room temperature magnetization switching in topological insulator-ferromagnet heterostructures by spin-orbit torques. Nat. Commun. 8, 1364 (2017).

18. Han, J. et al. Room-temperature spin-orbit torque switching induced by a topological insulator. Phys. Rev. Lett. 119, 077702 (2017).

19. Mahendra, D. C. et al. Room-temperature high spin-orbit torque due to quantum confinement in sputtered $\mathrm{Bi}_{\mathrm{x}} \mathrm{Se}_{(1-\mathrm{x})}$ films. Nat. Mat. 17, 800-807 (2018). 
20. Teo, J. C. Y., Fu, L. \& Kane, C. L. Surface states and topological invariants in three-dimensional topological insulators: Application to $\mathrm{Bi}_{1-\mathrm{x}} \mathrm{Sb}_{\mathrm{x}}$. Phys. Rev. B 78, 045426 (2008).

21. Ueda, Y., Khang, N. H. D., Yao, K. \& Hai, P. N. Epitaxial growth and characterization of $\mathrm{Bi}_{1-\mathrm{x}} \mathrm{Sb}_{\mathrm{x}}$ spin Hall thin films on GaAs(111) A substrates. Appl. Phys. Lett. 110, 062401 (2017).

22. Khang, N. H. D., Ueda, Y. \& Hai, P. N. A conductive topological insulator with large spin Hall effect for ultralow power spin-orbit torque switching. Nat. Mater. 17, 808-813 (2018).

23. Chi, Zh. D. et al. The spin Hall effect of Bi-Sb alloys driven by thermally excited Dirac-like electrons. Sci. Adv. 6, 2324 (2020).

24. Fan, T., Khang, N. H. D., Shirokura, T., Huy, H. H. \& Hai, P. N. Low power spin-orbit torque switching in sputtered BiSb topological insulator/perpendicularly magnetized CoPt/MgO multilayers on oxidized Si substrate. Appl. Phys. Lett. 119, 082403 (2021).

25. Roschewsky, N. et al. Spin-orbit torque and nernst effect in Bi-Sb/Co heterostructures. Phys. Rev. B 99, 195103 (2019).

26. Liu, E. et al. $\mathrm{Co} / \mathrm{Ni}-\mathrm{CoFeB}$ hybrid free layer stack materials for high density magnetic random access memory applications. Appl. Phys. Lett. 108, 3 (2016).

27. Fan, T., Tobah, M., Shirokura, T., Khang, N. H. D. \& Hai, P. N. Crystal growth and characterization of topological insulator BiSb thin films by sputtering deposition on sapphire substrates. Jpn. J. Appl. Phys. 59, 063001 (2020).

28. Gajek, M. et al. Spin torque switching of $20 \mathrm{~nm}$ magnetic tunnel junctions with perpendicular anisotropy. Appl. Phys. Lett. 100, 132408 (2012).

29. Sato, H. et al. Properties of magnetic tunnel junctions with a $\mathrm{MgO} / \mathrm{CoFeB} / \mathrm{Ta} / \mathrm{CoFeB} / \mathrm{MgO}$ recording structure down to junction diameter of $11 \mathrm{~nm}$. Appl. Phys. Lett. 105, 062403 (2014).

30. Hayashi, M., Kim, J., Yamanouchi, M. \& Ohno, H. Quantitative characterization of the spin-orbit torque using harmonic Hall voltage measurements. Phys. Rev. B 89, 144425 (2014).

31. Kim, J. et al. Layer thickness dependence of the current-induced effective field vector in Ta| CoFeB| MgO. Nat. Mater. 12, 240-245 (2013).

32. Shao, Q. et al. Strong Rashba-Edelstein effect-induced spin-orbit torques in monolayer transition metal dichalcogenide/ferromagnet bilayers. Nano Lett. 16, 7514-7520 (2016).

33. Wu, H. et al. Spin-orbit torque switching of a nearly compensated ferrimagnet by topological surface states. Adv. Mater. 31, 1901681 (2019).

34. Khang, N. H. D. \& Hai, P. N. Spin-orbit torque as a method for field-free detection of in-plane magnetization switching. Appl. Phys. Lett. 117, 252402 (2020).

35. Shirokura, T. \& Hai, P. N. Angle resolved second harmonic technique for precise evaluation of spin orbit torque in strong perpendicular magnetic anisotropy systems. Appl. Phys. Lett. 119, 222402 (2021).

36. Jinnai, B. et al. Spin-orbit torque induced magnetization switching in Co/Pt multilayers. Appl. Phys. Lett. 111, 102402 (2017).

37. Yasuda, K. et al. Current-nonlinear Hall effect and spin-orbit torque magnetization switching in a magnetic topological insulator. Phys. Rev. Lett. 119, 137204 (2017).

38. Koch, R. H., Katine, J. A. \& Sun, J. Z. Time-resolved reversal of spin-transfer switching in a nanomagnet. Phys. Rev. Lett. 92, 088302 (2004).

39. Sato, H. et al. $\mathrm{CoFeB}$ thickness dependence of thermal stability factor in $\mathrm{CoFeB} / \mathrm{MgO}$ perpendicular magnetic tunnel junctions. IEEE Magn. Lett. 3, 3000204 (2012).

40. Sato, H. et al. Perpendicular-anisotropy CoFeB-MgO magnetic tunnel junctions with a $\mathrm{MgO} / \mathrm{CoFeB} / \mathrm{Ta} / \mathrm{CoFeB} / \mathrm{MgO}$ recording structure. Appl. Phys. Lett. 101, 022414 (2012).

41. Li, X. et al. Materials requirements of high-speed and low-power spin-orbit-torque magnetic random-access memory. IEEE J. Electron Devices Soc. 8, 674-680 (2020).

42. Shiokawa, Y. et al. High write endurance up to $10^{12}$ cycles in a spin current-type magnetic memory array. AIP Adv. 9,035236 (2019).

43. Ryu, K.-S.S., Thomas, L., Yang, S.-H. \& Parkin, S. Chiral spin torque at magnetic domain walls. Nat. Nanotech. 8, 527-533 (2013).

44. Liu, L., Pai, C.-F., Ralph, D. C. \& Buhrman, R. A. Magnetic Oscillations Driven by the Spin Hall Effect in 3-Terminal Magnetic Tunnel Junction Devices. Phys. Rev. Lett. 109, 186602 (2012).

45. Shirokura, T. \& Hai, P. N. Bias-field-free spin Hall nano-oscillators with an out-of-plane precession mode. J. Appl. Phys. 127, 103904 (2020).

\section{Acknowledgements}

This work is supported by the CREST program of the Japan Science and Technology Agency (No. JPMJCR18T5). We sincerely thank N. Hatakeyama and H. Iida at the Material Analysis Division of Tokyo Institute of Technology for their help in TEM and XRD measurements.

\section{Author contributions}

T.F. fabricated the samples with contribution from N.H.D.K and S.N. T.F. performed spin-torque measurements. P.N.H supervised the project. T.F. and P.N.H wrote the paper with input from N.H.D.K and S.N.

\section{Competing interests}

The authors declare no competing interests.

\section{Additional information}

Supplementary Information The online version contains supplementary material available at https://doi.org/ 10.1038/s41598-022-06779-3.

Correspondence and requests for materials should be addressed to P.N.H.

Reprints and permissions information is available at www.nature.com/reprints.

Publisher's note Springer Nature remains neutral with regard to jurisdictional claims in published maps and institutional affiliations. 
(c) (i) Open Access This article is licensed under a Creative Commons Attribution 4.0 International cc) License, which permits use, sharing, adaptation, distribution and reproduction in any medium or format, as long as you give appropriate credit to the original author(s) and the source, provide a link to the Creative Commons licence, and indicate if changes were made. The images or other third party material in this article are included in the article's Creative Commons licence, unless indicated otherwise in a credit line to the material. If material is not included in the article's Creative Commons licence and your intended use is not permitted by statutory regulation or exceeds the permitted use, you will need to obtain permission directly from the copyright holder. To view a copy of this licence, visit http://creativecommons.org/licenses/by/4.0/.

(C) The Author(s) 2022 